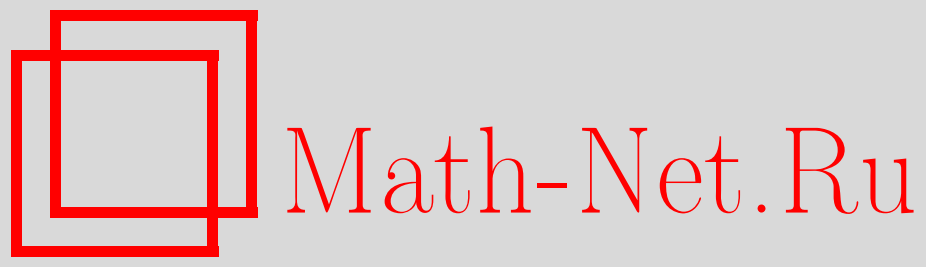

П. Б. Затицкий, О масштабирующей энтропийной последовательности динамической системы, Функи. анализ и его прил., 2014, том 48, выпуск $4,70-74$

DOI: https://doi.org/10.4213/faa3165

Использование Общероссийского математического портала MathNet.Ru подразумевает, что вы прочитали и согласны с пользовательским соглашением http://www.mathnet.ru/rus/agreement

Параметры загрузки:

IP : 3.81 .55 .215

26 апреля 2023 г., 16:38:41

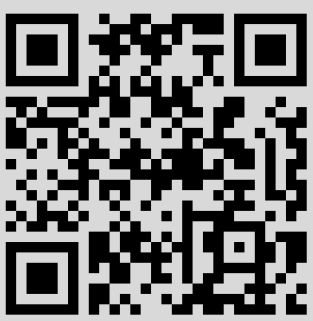


4, физ. хим., 1990, № 4, 78-82. [14] М. М. Фаддеев, Функц. анализ и его прил., 26:4 (1992), 80-83. [15] М. М. Фаддеев, Р. Г. Штеренберг, Зап. научн. сем. ПОМИ, 270 (2000), 336-349. [16] А. В. Киселев, М. М. Фаддеев, Функц. анализ и его прил., 34:2 (2000), 78-81. [17] М. М. Фаддеев, Р. Г. Штеренберг, Матем. заметки, 72:2 (2002), 292-302. [18] А. С. Костенко, Матем. заметки, 78:1 (2005), 147-151.

Universidad Autonoma del Estato de Hidalgo, Mexico e-mail: anauaeh@yahoo.com

Поступило в редакцию 15 июля 2013 г.

УДК 517.987.5

\title{
О масштабирующей энтропийной последовательности динамической системы*
}

\author{
(c) 2014. П. Б. ЗАтицкий
}

На протяжении работы все встречающиеся пространства с мерой будут стандартными вероятностными пространствами (пространствами Лебега-Рохлина). Основной интерес для нас будут представлять пространства с непрерывной мерой, однако все определения даются для произвольного пространства Лебега, т. е. пространства, в котором мера может содержать атомы. Говоря о вероятностном пространстве, мы позволим себе опускать обозначение сигма-алгебры.

1. Определение и свойства масштабирующей энтропийной последовательности. Напомним определение допустимой полуметрики и ее $\varepsilon$-энтропии (см., например, работы [11], [12]):

Определение 1. Полуметрику $\rho$ на стандартном вероятностном пространстве $(X, \mu)$ назовем измеримой, если она измерима на $\left(X^{2}, \mu^{2}\right)$ как функция двух переменных, и допустимой, если она сепарабельна на некотором подмножестве $X_{1} \subset X$ полной меры. В этих случаях тройку $(X, \mu, \rho)$ мы будем называть полуметрической и допустимой полуметрической соответственно.

Следующее определение восходит к А. Н. Колмогорову.

Определение 2. Для каждого $\varepsilon>0$-энтропия $\mathbb{H}_{\varepsilon}(X, \mu, \rho)$ полуметрической тройки $(X, \mu, \rho)$ определяется по следующему правилу. Рассмотрим наименьшее натуральное $k$, для которого пространство $X$ можно представить в виде объединения множеств $X_{0}, X_{1}, \ldots, X_{k}$, таких, что $\mu\left(X_{0}\right)<\varepsilon$ и при $j=$ $1, \ldots, k$ диаметр множества $X_{j}$ в полуметрике $\rho$ меньше $\varepsilon$. Положим

$$
\mathbb{H}_{\varepsilon}(X, \mu, \rho)=\log _{2} k .
$$

Если такого $k$ не существует, положим $\mathbb{H}_{\varepsilon}(X, \mu, \rho)=+\infty$. В то же время $\varepsilon$-энтропией называют и функцию $\varepsilon \mapsto \mathbb{H}_{\varepsilon}(X, \mu, \rho)$

Напомним, что измеримая полуметрика $\rho$ на стандартном вероятностном пространстве $(X, \mu)$ допустима тогда и только тогда, когда $\mathbb{H}_{\varepsilon}(X, \mu, \rho)<+\infty$ для любого $\varepsilon>0$ (подробности см. в работе [12]).

*Работа выполнена при поддержке Лаборатории им. П. Л. Чебышева СПбГУ (грант Правительства РФ, дог. 11.G34.31.0026), ОАО «Газпром нефть», гранта СПбГУ 6.38.223.2014, грантов РФФИ 13-01-12422 офи_м2 и 14-01-00373_А, гранта Президента РФ МК-6133.2013.1. 
Далее мы считаем преобразование $T$ эргодическим автоморфизмом стандартного вероятностного пространства $(X, \mu)$. Пусть $\rho$ - измеримая полуметрика на $(X, \mu)$. Сдвиги $T^{k} \rho(x, y)=\rho\left(T^{k} x, T^{k} y\right)$, очевидно, также являются измеримыми полуметриками на $(X, \mu)$, причем для любого $\varepsilon>0$ выполнено равенство

$$
\mathbb{H}_{\varepsilon}(X, \mu, \rho)=\mathbb{H}_{\varepsilon}\left(X, \mu, T^{k} \rho\right) .
$$

Определим конечные усреднения полуметрики $\rho$ под действием оператора $T$ формулой $T_{a v}^{n} \rho=\frac{1}{n} \sum_{k=0}^{n-1} T^{k} \rho$.

Важным динамическим свойством полуметрик является следующее свойство.

Определение 3. Измеримая на $(X, \mu)$ полуметрика $\rho$ называется (двусторонней) порождающей для метрической динамической системы $(X, \mu, T)$, если ее сдвиги $T^{k} \rho, k \in \mathbb{Z}$, в совокупности разделяют точки с точностью до множества меры нуль, т. е. существует некоторое множество $X^{\prime} \subset X$ полной меры, такое, что для любых различных $x, y \in X^{\prime}$ найдется $k \in \mathbb{Z}$, такое, что $T^{k} \rho(x, y)>0$.

Отметим, что допустимая метрика является порождающей для любого автоморфизма $T$, так как уже она разделяет точки.

Напомним определение, данное А. М. Вершиком в работах [7], [9], [10].

Определение 4. Пусть $(X, \mu, T)$ - метрическая динамическая система, а $\rho$ - допустимая полуметрика на $(X, \mu)$. Последовательность положительных чисел $\left\{h_{n}\right\}$ называется масштабирующей для $\rho$, если для любого $\varepsilon>0$ выполнено неравенство

$$
\varlimsup_{n} \frac{\mathbb{H}_{\varepsilon}\left(X, \mu, T_{a v}^{n} \rho\right)}{h_{n}}<+\infty
$$

а при достаточно малых $\varepsilon>0-$ неравенство

$$
0<\underline{\lim } \frac{\mathbb{H}_{\varepsilon}\left(X, \mu, T_{a v}^{n} \rho\right)}{h_{n}} .
$$

Класс всех масштабирующих последовательностей для полуметрики $\rho$ обозначим символом $\mathcal{H}(X, \mu, T, \rho)$.

Замечание 1. Если $\rho$ - допустимая полуметрика, $h=\left\{h_{n}\right\} \in \mathcal{H}(X, \mu, T, \rho)$, а $h^{\prime}=\left\{h_{n}^{\prime}\right\}$ - последовательность положительных чисел, то $h^{\prime} \in \mathcal{H}(X, \mu, T, \rho)$ тогда и только тогда, когда $0<\underline{\lim } h_{n}^{\prime} / h_{n} \leqslant \varlimsup \lim h_{n}^{\prime} / h_{n}<\infty$.

Следующая теорема подтверждает гипотезу Вершика о независимости масштабирующей последовательности от полуметрики, выдвинутую в работе [10]. Для автоморфизмов с чисто точечным спектром она была доказана в работе [12] (см. теорему 4 далее).

Теорема 1. Пусть $\rho, \tilde{\rho}-$ суммируемые допустимые порождающие полуметрики на $(X, \mu)$. Тогда $\mathcal{H}(X, \mu, T, \rho)=\mathcal{H}(X, \mu, T, \tilde{\rho})$.

Теорема 1 приводит к следующему определению.

Определение 5. Последовательность $h=\left\{h_{n}\right\}$ положительных чисел называется масштабирующей энтропийной последовательностъю метрической динамической системы $(X, \mu, T)$, если $h \in \mathcal{H}(X, \mu, T, \rho)$ для некоторой (а тогда и любой) суммируемой допустимой порождающей полуметрики $\rho$. Класс масштабирующих энтропийных последовательностей динамической системы обозначим через $\mathcal{H}(X, \mu, T)$. 
Следствие 1. Класс масштабирующих энтропийнъх последовательностей является метрическим инвариантом эргодических динамических систем.

Автору до сих пор не известно, для любой ли динамической системы существует масштабирующая энтропийная последовательность (т. е. множество $\mathcal{H}(X, \mu, T)$ непусто).

Класс масштабирующих энтропийных последовательностей монотонен в следующем смысле:

Следствие 2. Пусть динамическая система $\left(X_{2}, \mu_{2}, T_{2}\right)$ является фактором системы $\left(X_{1}, \mu_{1}, T_{1}\right)$, a $h^{1}=\left\{h_{n}^{1}\right\}, h^{2}=\left\{h_{n}^{2}\right\}-$ их масштабирующие энтропийные последовательности соответственно. Тогда $h_{n}^{2}=O\left(h_{n}^{1}\right), n \rightarrow+\infty$.

2. Примеры. 2.1. Связъ с колмогоровской энтропией автоморфизма. Следующая теорема проясняет связь между масштабирующими энтропийными последовательностями и энтропией Колмогорова.

Теорема 2. Пусть $T$ - эргодический автоморфизм пространства $(X, \mu)$. Тогда:

(1) если колмогоровская энтропия $h_{\mu}(T)$ конечна и положительна, то последовательность $h_{n}=n$ является масштабирующей энтропийной последовательностью системы $(X, \mu, T)$;

(2) если $h_{n}$ - масштабирующая энтропийная последовательность системы $(X, \mu, T)$, то $h_{\mu}(T)=0$ тогда и только тогда, когда $h_{n}=o(n), n \rightarrow+\infty$.

Сравнительно просто вычисляется масштабирующая энтропийная последовательность сдвига Бернулли. Пусть $(A, \nu)$ - стандартное вероятностное пространство, $X=A^{\mathbb{Z}}$ - пространство двусторонних последовательностей, $\mu=\nu^{\mathbb{Z}}$ - продакт-мера на $X$, а $T: X \rightarrow X$ - левый сдвиг.

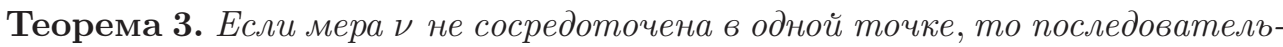
ность $h_{n}=n$ является масштабирующей энтропийной последовательностью для сдвига Бернулли $(X, \mu, T)$.

Если мера $\nu$ непрерывна, то энтропия пространства $(A, \nu)$ бесконечна. При этом в соответствии с теоремой Колмогорова (см. [1]-[3]) колмогоровская энтропия $h_{\mu}(T)$ будет бесконечной. Таким образом, обращение утверждения (1) теоремы 2 неверно.

2.2. Автоморфизмы с чисто точечным спектром. Ранее в работе [12] была доказана следующая теорема, характеризующая автоморфизмы с чисто точечным спектром в терминах масштабирующих энтропийных последовательностей.

Теорема 4. Пусть $T$ - эргодический автоморфизм стандартного вероятностного пространства $(X, \mu)$. Тогда равносильны следующие утверждения:

(1) спектр системы $(X, \mu, T)$ чисто точечный;

(2) множество $\mathcal{H}(X, \mu, T)$ состоит из положительных ограниченных отделенных от нуля последовательностей.

Сравним этот результат с другим критерием энтропийного типа чистой точечности спектра, полученным Кушниренко в работе [4]. Напомним определение $A$-энтропии (последовательностной энтропии) эргодического автоморфизма $T$ стандартного вероятностного пространства $(X, \mu)$. Для возрастающей последовательности натуральных чисел $A=\left\{a_{n}\right\}$ и разбиения $\xi$ пространства $(X, \mu)$ с конечной энтропией определяется число $h_{A}(T, \xi)=\varlimsup_{n}\left(H\left(\bigcap_{i=1}^{n} T^{a_{i}} \xi\right) / n\right)$, где 
$H$ - энтропия измеримого разбиения. $A$-энтропией автоморфизма $T$ называется число $h_{A}(T)=\sup _{\xi} h_{A}(T, \xi)$, где супремум берется по всем измеримым разбиениям с конечной энтропией. В работе [4] доказано, что спектр автоморфизма $T$ является чисто точечным тогда и только тогда, когда $h_{A}(T)=0$ для любой последовательности $A$. Сравнивая этот результат с теоремой 4 , получаем, что если $h_{A}(T)>0$, то масштабирующая энтропийная последовательность растет. Следующая теорема дает количественную оценку этого утверждения.

Теорема 5. Если $A=\left\{a_{n}\right\}, h_{A}(T)>0, a h=\left\{h_{n}\right\}$ - масштабирующая энтропийная последовательность автоморфизма $T$, mо $\underline{\lim }\left(h_{a_{n}} / \log \log n\right)>0$.

2.3. Подстановки постоянной длины. В качестве примера продемонстрируем вычисление масштабирующих энтропийных последовательностей подстановочных динамических систем постоянной длины. Необходимые сведения из теории подстановочных динамических систем см., например, в монографии [8].

Пусть $\xi$ - подстановка длины $q$ на алфавите $A$ (т. е. отображение из $A$ в $A^{q}$, продолженное покоординатно до отображения конечных и бесконечных слов). Мы предполагаем, что для некоторого символа $\alpha \in A$ слово $\xi(\alpha)$ начинается с символа $\alpha$. В таком случае найдется бесконечное слово $u \in A^{\mathbb{N}_{0}}$ (где $\left.\mathbb{N}_{0}=\mathbb{N} \cup\{0\}\right)$, начинающееся с $\alpha$, такое, что $\xi(u)=u$. Пусть $T-$ левый сдвиг на $A^{\mathbb{N}_{0}}$, а $X_{\xi}$ - замыкание последовательности $T^{n} u, n \in \mathbb{N}$, в топологии произведения. Если подстановка $\xi$ примитивна, то топологическая динамическая система $\left(X_{\xi}, T\right)$ минимальна и, более того, обладает единственной инвариантной мерой $\mu^{\xi}$. Таким образом, примитивной подстановке соответствует метрическая динамическая система $\left(X_{\xi}, \mu^{\xi}, T\right)$. Оказывается, что масштабирующая энтропийная последовательность этой динамической системы может быть выражена в терминах двух комбинаторных параметров подстановки. Первый из них, высота $h(\xi)$, определяется, например, как наибольшее число $k$, взаимно простое с $q$, такое, что если $u_{n}=u_{0}$ (где $v_{m}$ для слова $v$ и числа $m$ - символ алфавита $A$, стоящий в слове $v$ на $m$-м месте), то $k \mid n$. Второй параметр, столбцовое число (column number) $c(\xi)$, определяется как $\min \left\{\left|\left\{\xi^{n}(a)_{k}: a \in A\right\}\right|: n \in \mathbb{N}, 0 \leqslant k<q^{n}\right\}$ (отметим, что наше определение немного отличается от классического, приведенного в работах [6] и [8]).

Теорема 6. Пусть $\xi-$ инвективная примитивная подстановка постоянной длины на алфавите $A,|A|>1$. Тогда последовательность $h_{n}=1+(\mathrm{c}(\xi)-$ $h(\xi)) \log n$ является масштабирующей энтропийной последовательностью динамической системы $\left(X_{\xi}, \mu^{\xi}, T\right)$.

Идея доказательства этой теоремы заключается в изучении свойств полуметрик на алфавите $A$, инвариантных относительно подстановки.

Из теорем 6 и 4 вытекает

Следствие 3. Пусть $\xi-$ - ивективная примитивная подстановка постоянной длины на алфавите $A,|A|>1$. Тогда спектр соответствующей ей динамической системы $\left(X_{\xi}, \mu^{\xi}, T\right)$ является чисто точечным в том и только том случае, когда $c(\xi)=h(\xi)$.

Отметим, что похожий критерий впервые был получен в работах [5] и [6], однако там он сформулирован несколько иначе. Отличие заключается в том, что для подстановок, высота которых больше единицы, параметр $c(\xi)$ определяется не так, как у нас, а путем рассмотрения некоторой модифицированной 
подстановки. Для случая $h(\xi)=1$ результат следствия 3 совпадает с критерием из этих работ - спектр является чисто точечным тогда и только тогда, когда $c(\xi)=1$. А для случая $h(\xi)>1$ критерий из следствия 3 , в отличие от критерия из этих работ, не требует перехода к модифицированной подстановке.

Автор выражает благодарность Ф. В. Петрову и А. М. Вершику за многочисленные полезные обсуждения, указания и помощь в работе.

\section{ЛитеРАТУРА}

[1] А. Н. Колмогоров, Докл. АН СССР, 119:5 (1958), 861-864. [2] А. Н. Колмогоров, Докл. АН СССР, 124 (1959), 754-755. [3] Я. Г. Синай, Докл. АН СССР, 124 (1959), 768-771. [4] А. Г. Кушниренко, УМН, 22:5(137) (1967), 57-65. [5] Т. Kamae, J. Math. Soc. Japan, 24 (1972), 285-306. [6] F. M. Dekking, Z. Wahrscheinlichkeitstheorie und Verw. Gebiete, 41:3 (1977/78), 221-239. [7] А. М. Вершик, А. Д. Горбульский, ТВП, 52:3 (2007), 446-467. [8] M. Queffélec, Substitution Dynamical Systems-Spectral Analysis, Lecture Notes in Math., Springer-Verlag, Berlin, 2010. [9] A. Vershik, Markov Processes and Related Fields, 16:1 (2010), 169-185. [10] А. М. Вершик, Алгебра и анализ, 23:1 (2011), 111-135. [11] П. Б. Затицкий, Ф. В. Петров, Зап. научн. сем. ПОМИ, 390 (2011), 201-209. [12] A. M. Vershik, P. B. Zatitskiy, F. V. Petrov, Cent. Eur. J. Math., 11:3 (2013), 379-400.

Лаборатория им. П. Л. Чебышева

Санкт-Петербургского государственного университета

Поступило в редакцию Санкт-Петербургское отделение Математического

института им. В. А. Стеклова РАН

e-mail: paxa239@yandex.ru

УДК $517.95+517.984 .5$

\section{Двумерные потенциалы Вигнера-фон Неймана с кратным положительным собственным значением*}

(C) 2014. Р. Г. НовИКОВ, И. А. ТАЙМАНов, С. П. ЦАРЕВ

Пусть $H$ - оператор Шрёдингера,

$$
H=-\Delta+U,
$$

с убывающим на бесконечности потенциалом $U(x)$ в $\mathbb{R}^{N}$. Потенциал $U$ называется потенииалом Вигнера-фон Неймана, если $H$ имеет положительное собственное значение с собственной функцией, принадлежащей $L_{2}\left(\mathbb{R}^{N}\right)$, т. е. существует точка его дискретного спектра, вложенная в абсолютно непрерывный спектр.

*Работа была частично поддержана ФЦП №14.А18.21.0866 Министерства образования и науки РФ (Р.Г.Н.), междисциплинарным проектом «Геометрические и алгебраические методы нахождения явных решений уравнений математической физики и механики сплошной среды» СО РАН (И.А.Т. и С. П. Ц.) и грантом 1431/ГФ Министерства образования и науки Республики Казахстан (И. А. Т.). Она также получила поддержку в рамках государственного задания Министерства образования и науки по Сибирскому федеральному университету на выполнение НИР в 2014 году, задание №1.1462.2014/К (С.П.Ц.). 